\title{
DOENÇAS DERMATOLÓGICAS FREQUENTES EM UNIDADE BÁSICA DE SAÚDE
}

\author{
Kamilla Maestá Agostinhoํ, Karenine Maria Holanda Cavalcante², Pacífica Pinheiro Cavalcanti ${ }^{3}$,
} Débora Linsbinski Pereira ${ }^{4}$

RESUMO: Objetivou-se identificarpatologias dermatológicas mais frequentes no Bairro BoaEsperançadacidade de Sinop-Mato Grosso, e descrever dados clínicos e demográficos das pessoas acometidas. Pesquisa descritiva, quantitativa, documental, desenvolvida pormeio da coleta de dados nos prontuários dos pacientes, entre agosto e setembro de 2010. Dos 196 usuários com diagnóstico de doença dermatológica, 107(54,6\%) eram mulherese $55 \%$ crianças entre 0 a 5 anos de idade. As patologias mais frequentes foram o impetigo 14,3\%; Varicela 11,7\%; Escabiose 8,2\%; Dermatite de contato, 7,6\% e Tínea corpus, 6,6\%. Grande parte dessas doenças são preveníveis por meio de medidas de educação em saúde e saneamento básico. O enfermeiro encontra-se em posição privilegiada na saúde coletiva, podendo proporcionar diagnóstico precoce e prevenção de patologias dermatológicas por meio da realização de exame físico e de medidas de educação em saúde. DESCRITORES: Dermatologia; Prevalência; Pele; Enfermagem.

\section{FREQUENT DERMATOLOGICAL DISEASES IN PRIMARY HEALTH CARE CENTERS}

ABSTRACT: The aim was to identify the most frequent dermatological pathologies in the neighborhood of Boa Esperança in the city of Sinop in the state of Mato Grosso, and to describe the clinical and demographic data of the persons affected. This is descriptive, quantitative, documental research, undertaken through the collection of data from the patients' hospital records between August and September 2010. Of the 196 service users with a diagnosis of a dermatological disease, $107(54.6 \%)$ were women and 55\% children aged between 0 and 5 years of age. The most frequent pathologies were impetigo 14.3\%; Varicella 11.7\%; Scabies 8.2\%; Contact dermatitis, 7.6\% and Ringworm, 6.6\%. A large proportion of these diseases are preventable through health education measures and basic sanitation. The nurse is in a privileged position in public health, being able to provide early diagnosis and prevention of dermatological pathologies by undertaking physical tests and providing health education measures. DESCRIPTORS: Dermatology; Prevalence; Skin; Nursing.

\section{ENFERMEDADES DERMATOLÓGICAS FRECUENTES EN UNIDAD BÁSICA DE SALUD}

RESUMEN: Fue objetivo de este estudio identificar patologías dermatológicas más frecuentes en el barrio Boa Esperança de la ciudad de Sinop - Mato Grosso, así como describir datos clínicos y demográficos de las personas afligidas. Investigación descriptiva, cuantitativa, documental, desarrollada por medio de datos obtenidos en los prontuarios de los pacientes, entre agosto y septiembre de 2010. De los 196 usuarios con diagnóstico de enfermedad dermatológica, 107(54,6\%) eran mujeres y $55 \%$ niños entre 0 y 5 años de edad. Las patologías más frecuentes fueron la enfermedad causada por la bacteria Staphylococcus aureus o Streptococcus pyogenes 14,3\%; varicela $11,7 \%$; sarna 8,2\%; dermatitis de contacto, 7,6\% y Tínea corpus, 6,6\%. Grande parte de esas enfermedades son prevenibles por medio de medidas de educación en salud y saneamiento básico. El enfermero está en posición privilegiada en la salud colectiva, siendo posible proporcionar diagnóstico precoz y prevención de patologías dermatológicas por medio de la realización de examen físico y de medidas de educación en salud.

DESCRIPTORES: Dermatología; Prevalencia; Piel; Enfermería.

${ }^{1}$ Enfermeira. Especialista em Saúde Pública. Professora da Universidade Federal de Mato Grosso - UFMT, Campus de Sinop.

${ }^{2}$ Enfermeira. Mestre em Enfermagem. Professora da Universidade Federal de Sergipe - Campus de Lagarto.

${ }^{3}$ Enfermeira. Doutora em Enfermagem. Professora da UFMT - Campus de Sinop.

${ }^{4}$ Enfermeira e farmacêutica. Mestranda em Ciências Ambientais pela UFMT. 


\section{INTRODUÇÃO}

A pele é o maior órgão do corpo e é indispensável para o ser humano, ela forma uma barreira entre os órgãos e o meio externo e ainda participa de funções vitais ao organismo. As estruturas que compõem o tegumento protegem o corpo contra lesões ambientais, auxiliam na regulação da temperatura corpórea, servem como órgãos sensoriais e facilitam a síntese de vitamina $\mathrm{D}^{(1)}$. É através da pele que são expressos inúmeros sinais e sintomas que são de auxílio para a identificação de doenças. Portanto, manifestações clínicas neste órgão podem ser relativas a patologias restritas à pele ou podem representar primeiros sinais de outras doenças ou mesmo manifestações tardias de patologias.

A história da Dermatologia moderna iniciou-se na Europa, em especial na França, entre os séculos XV e $\mathrm{XVI}$, onde médicos começam a se interessar por problemas cutâneos. No Brasil, a Dermatologia teve seu início em 1882, com a instalação do primeiro Serviço Clínico de Doenças da Pele na Policlínica Geral do Rio de Janeiro. A partir das descobertas da microbiologia, em finais do século XIX e início do século XX, e da dinâmica induzida pelo ensino da Dermatologia como especialidade, os estudos nesta área evoluíram ${ }^{(2)}$.

A Sociedade Brasileira de Dermatologia coloca que as doenças de pele figuram entre as três primeiras causas de demanda aos serviços de saúde. Entre as 25 causas mais frequentes em 2006, aquelas com maiores proporções de consulta de retorno foram hanseníase (78\%), psoríase $(68 \%)$ e vitiligo (64\%). Na Suécia a prevalência das doenças de pele está em $20,5 \%$, sendo $23,3 \%$ nas mulheres de $17,5 \%$ nos homens ${ }^{(3)}$. Nos Estados Unidos as queixas relacionadas à pele correspondem de 5 a $10 \%$ das consultas ambulatoriais. Comumente, o estresse psicológico devido a doença ou a vários problemas pessoais e familiares pode ser exibido externamente como problemas dermatológicos ${ }^{(4)}$.

Estudos mostram que as doenças dermatológicas têm significativo impacto na qualidade de vida dos atingidos, principalmente os cronicamente doentes, ressaltando a necessidade de sua valorização como problema de saúde pelos responsáveis pela formulação de políticas pública. Pessoas com doença dermatológica costumam perceber sua saúde alterada, podem sentir-se limitados na execução de suas tarefas diárias e experimentar perda de vitalidade. As doenças dermatológicas são, portanto, limitantes, provocam absenteísmo escolar e no trabalho, e seus portadores estão mais propensos a apresentar depressão ${ }^{(3,5)}$.
Muitas vezes, as patologias dermatológicas são consideradas um problema essencialmente estético. Entretanto, comumente tais doenças afetam amplamente os aspectos sociais e psicológicos da pessoa acometida, pois estas convivem com situações constrangedoras e preconceituosas que podem interferir em sua autoestima e na vida social.

O presente estudo visou identificar as doenças dermatológicas mais frequentes nos prontuários dos pacientes atendidos pela Unidade de Saúde da Família (USF) do Bairro Boa Esperança da cidade de Sinop Mato Grosso, que realizaram a primeira consulta entre julho de 2009 e julho de 2010, bem como a descrever o perfil demográfico e clínico dos pacientes.

Ressalta-se que este estudo considera prontuário do paciente como um registro de informações, sinais e imagens referentes ao paciente, baseada em fatos e situações sobre a saúde e assistência prestada ao indivíduo, que possibilita, entre outras funções, a comunicação entre membros de equipe multiprofissional, o ensino e a pesquisa em saúde ${ }^{(6)}$. Portanto, as informações contidas nos prontuários dos pacientes estão acessíveis aos profissionais da equipe multiprofissional da saúde, permitindo a continuidade da assistência prestada. Assim, qualquer falha no registro dos dados pode trazer prejuízos na assistência ao cliente proporcionada por toda a equipe de saúde.

Destaca-se, ainda, que esta pesquisa não visa avaliar a atuação de profissionais da saúde responsáveis por registro de informações no prontuário do paciente. Busca-se, apenas discutir as informações contidas nos prontuários de modo a caracterizar a população estudada.

\section{MÉTODO}

Este trabalho caracteriza-se como pesquisa aplicada, quantitativa, descritiva e documental, pois é elaborada a partir de materiais que ainda não receberam tratamento analítico. Determinou-se como população prontuários de pessoas com doenças dermatológicas atendidas entre julho de 2009 e julho de 2010 na USF localizado no Bairro Boas Esperança e residentes no mesmo bairro, do município de $\mathrm{Si}$ nop - Mato Grosso. Foram incluídos prontuários de pessoas em qualquer faixa etária, sexo, com ou sem patologia associada, totalizando 106 prontuários.

A coleta dos dados foi realizada no período de agosto a setembro de 2010 com auxílio de um instrumento contendo as seguintes variáveis: nome, data de nascimento, sexo, raça, patologia dermatológica, 
sinais e sintomas, tratamento e tempo do tratamento. Contudo, enfrentou-se grande dificuldade na coleta das informações devido à considerável falta de dados registrados; desse modo, dados como raça e tempo de tratamento não foram coletados.

Os dados referentes à variável patologia dermatológica foram extraídos do prontuário do paciente no espaço destinado à expressão do diagnóstico médico, entretanto, este estudo não visa discutir o processo diagnóstico médico; e sim, discutir as patologias mais frequentes, considerando o conceito de patologia como o estado de saúde anormal ou desviante ${ }^{(7)}$ e, neste caso, as que afetam o tegumento humano. É importante ressaltar que em muitos prontuários não foi encontrado o registro do diagnóstico médico, mas sim, sinais relacionados às doenças tegumentares. Nesse caso, optou-se por incluir na tabela da mesma forma que estava registrada no prontuário, ou seja, como sinal da doença.

Os dados foram analisados por meio de estatística descritiva e organizados em tabela. A pesquisa respeitou os preceitos éticos descritos na Resolução 196/96 determinada pelo Conselho Nacional de Saúde ${ }^{(8)}$, com destaque aos quatro referenciais básicos da bioética: autonomia, não maleficência, beneficência e justiça. $\mathrm{O}$ estudo foi desenvolvido após ser analisado e deliberado pelo Comitê de Ética em Pesquisa com Seres Humanos (protocolo n. 810/CEP-HUJM/10).

\section{RESULTADOS}

Essa seção expõe de maneira descritiva, por meio de frequência absoluta e relativa, os dados encontrados acerca das cinco doenças dermatológicas mais comuns. Dos 196 prontuários de pacientes com patologia dermatológica, 107(54,6\%) eram pessoas do sexo feminino e $89(45,4 \%)$ do sexo masculino. Com relação à faixa etária $108(55,1 \%)$ eram crianças ( $0-9$ anos), 22(11,2\%) adolescentes (10-19 anos), 11(5,6\%) adultos jovens (2023 anos), 48(24,5\%) adultos (24-59 anos) e 7(3,6\%) eram idosos (60 anos ou mais).

A tabela 1 inclui todas as patologias identificadas na pesquisa.

As cinco patologias mais comuns nesta unidade de saúde foram observadas em 95 prontuários (48,5\%). Quanto à faixa etária das pessoas acometidas pelo Impetigo, prevaleceram crianças entre nove meses e nove anos, perfazendo $25(89,3 \%)$ prontuários dos 28 , envolvendo pessoas de até 75 anos. Nesta variável, a moda foi de um e dois anos, que envolveu seis prontuários cada.
Tabela 1 - Patologias tegumentares identificadas. Sinop, 2009-2010

\begin{tabular}{|c|c|c|c|}
\hline Patologia & $\mathbf{N}$ & Mulher & Homem \\
\hline$\overline{\text { Impetigo }}$ & 28 & 16 & 12 \\
\hline Varicela & 23 & 14 & 09 \\
\hline Escabiose & 16 & 08 & 08 \\
\hline Dermatite de contato & 15 & 08 & 07 \\
\hline Tínea corpus & 13 & 08 & 05 \\
\hline Ptiríase versicolor & 11 & 07 & 04 \\
\hline $\begin{array}{l}\text { Manchas hipocrô- } \\
\text { micas, descamativas }\end{array}$ & 11 & 06 & 05 \\
\hline Hanseníase & 09 & 03 & 06 \\
\hline Micose & 08 & 03 & 05 \\
\hline Dermatite de fraldas & 07 & 04 & 03 \\
\hline Candidíase oral & 05 & 02 & 03 \\
\hline Candidíase vaginal & 05 & 05 & 00 \\
\hline Larva migrans & 05 & 03 & 02 \\
\hline Mancha & 05 & 05 & 00 \\
\hline Piodermite & 04 & 00 & 04 \\
\hline Furunculose & 03 & 01 & 02 \\
\hline Eczema & 03 & 02 & 01 \\
\hline Nevus & 03 & 01 & 02 \\
\hline $\begin{array}{l}\text { Molusco } \\
\text { contagioso }\end{array}$ & 03 & 00 & 03 \\
\hline $\begin{array}{l}\text { Leishmaniose } \\
\text { cutânea }\end{array}$ & 02 & 00 & 02 \\
\hline Alopécia & 02 & 01 & 01 \\
\hline Erisipela & 02 & 02 & 00 \\
\hline $\begin{array}{l}\text { Dermatite } \\
\text { seborréica }\end{array}$ & 02 & 02 & 00 \\
\hline Vitiligo & 02 & 02 & 00 \\
\hline Dermatite atópica & 01 & 00 & 01 \\
\hline Psoríase & 01 & 00 & 01 \\
\hline Dermatose & 01 & 00 & 01 \\
\hline Tínea pedis & 01 & 01 & 00 \\
\hline Ptiríase Alba & 01 & 00 & 01 \\
\hline Acne & 01 & 01 & 00 \\
\hline Lesão & 01 & 01 & 00 \\
\hline Herpes & 01 & 01 & 00 \\
\hline Urticária recorrente & 01 & 01 & 00 \\
\hline
\end{tabular}

Em relação aos tratamentos mais prescritos para a cura do Impetigo, destacou-se o permanganato de potássio $(n=16 ; 59,2 \%)$ dos 27 tratamentos; em um não havia tratamento prescrito. Outros medicamentos utilizados foram: cefalexina, antibiótico, nebacetin, benzetacil, neomicina, amoxicilina, vancomicina, paracetamol, miconazol, nistatina e metronidazol.

No que se refere à Varicela, a faixa etária mais afetada foi de crianças com idade entre quatro me- 
ses e oito anos, com 17(73,9\%) prontuários dos 23 identificados com essa patologia; a variação da idade nesta patologia foi de quatro meses a 35 anos. Nesta variável, a moda foi de oito anos e o sexo feminino foi o mais acometido, com 14(60,9\%) pessoas. E para o tratamento da Varicela, a pasta d'água foi presente em $17(85 \%)$ dos 20 tratamentos prescritos. Também foram identificados associação com zovirox ${ }^{\circledR}$ pomada, paracetamol ${ }^{\circledR}$, polaradex ${ }^{\circledR}$, pergamanato de potássio, amoxicilina e cefalexina..

A Escabiose manifestou-se com maior prevalência em crianças na faixa etária entre quatro meses a sete anos, aparecendo em nove (56,2\%) prontuários dos 16 , atingindo pessoas com até a idade de 54 anos; a moda foi de sete anos e ambos os sexos $(\mathrm{n}=8 ; 50 \%)$ foram acometidos igualmente. Referindo-se ao tratamento prescrito, o benzoato de benzila foi indicado para nove $(60 \%)$ dos 15 pacientes, entretanto, em um prontuário não havia tratamento prescrito. Além deste medicamento, foram prescritos dexametasona ${ }^{\circledR}$ creme, polaradex ${ }^{\circledR}$, vermectil $\AA$, amoxicilina, neomicina $\AA$, miconazol $\AA$, paracetamol $\AA$, loratadina $\AA$, prometazina ${ }^{\circledR}$, permetrina $\AA$ solução, ivermectina ${ }^{\circledR}$ e dermatil ${ }^{\circledR}$ como tratamento.

A Dermatite de Contato esteve presente com maior frequência em crianças na faixa etária entre 20 dias e oito anos, com nove (60\%) prontuários de 15 , sendo que a variação da idades foi de 20 dias a 37 anos. A Dermatite acometeu mais o sexo feminino, com oito(53,3\%) pessoas. Para o tratamento da Dermatite de Contato, a dexametasona ${ }^{\circledR}$ aparece em quatro $(36,4 \%)$ dos 11 tratamentos prescritos, sendo que em quatro prontuários não existia tratamento indicado. Outros tratamentos encontrados foram dermacyd ${ }^{\circledR}$ liquido, pergamanato de potássio, cetoconazol ${ }^{\circledR}$, neomicina ${ }^{\circledR}$, novacort ${ }^{\circledR}$ pomada, cefalexina, dermatop ${ }^{\circledR}$ creme e polaradex ${ }^{\circledR}$.

No que concerne à Tínea corpus houve variação dentro da faixa etária de um a 54 anos, sendo que não foi possível identificar a moda, pois acometeu diferentes idades uma única vez nos 13 prontuários. Entre as pessoas afetadas com Tínea corpus o sexo feminino foi o mais afetado, com oito(61,5\%) pacientes. O cetoconazol ${ }^{\circledR}$ pomada aparece em $08(61,5 \%)$ dos 13 tratamentos prescritos. Neste caso, todos possuíam o registro do tratamento, sendo, portanto, outros medicamentos prescritos miconazol $\AA$, dexametasona ${ }^{\circledR}$, cefalexina $\AA$, neomicina ${ }^{\circledR}$, metronidazol ${ }^{\circledR}$, mebendazol ${ }^{\circledR}$, fluconazol ${ }^{\circledR}$ e micofim $\AA$ creme.

Em relação ao mês (entre julho de 2009 a julho de 2010) em que as cinco patologias mais comuns ocorreram com maior frequência foram Impetigo, em maio de
2010, sete(25\%) prontuários; Varicela, em setembro de 2009, 10(43,5\%) prontuários; Escabiose, em fevereiro de 2010, quatro(25\%) prontuários; Dermatite de Contato, em julho de 2010, quatro(26,7\%) prontuários; e Tínea corpus, em janeiro de 2010, três(20,1\%) prontuários.

\section{DISCUSSÃO}

As mulheres foram as mais acometidas por doenças dermatológicas com 107(54,6\%) atendimentos, contra $89(45,4 \%)$ atendimentos a homens, o que corresponde a uma relação de 1,2:1. As mulheres são as que mais procuram serviços de saúde no Brasil, sejam para atendimento pessoal ou para seus filhos ${ }^{(9)}$. Nas relações socioculturais, que homens e mulheres estabelecem, assinala-se que a ideia de cuidados com a saúde é associada à fragilidade, e os serviços de saúde costumam ser identificados como locais de mulheres, crianças e idosos ${ }^{(10)}$.

Pode-se supor, ainda, que, no Brasil, as mulheres procuram em maior número o atendimento dermatológico por serem, de modo geral, mais atenciosas com o corpo e vaidosas, característica comumente feminina. As mulheres brasileiras foram descritas como as mais vaidosas do mundo. Esta atenção é extensiva para seus filhos e filhas ${ }^{(11)}$. Considerando as características femininas, as mulheres costumam ter maior afinidade com a habilidade de cuidar de pessoas, nos mais diversos aspectos do ser humano. Neste estudo $55 \%$ dos atendimentos foram com crianças entre 0 a 5 anos de idade, fato este relacionado com a preocupação das mães com a saúde de suas filhas e filhos, às vezes, no entanto, um cuidado maior com eles do que consigo mesmo; mas também, especialmente, por forte vínculo da maternidade, gerando procura imediata por assistência diante de qualquer alteração na saúde deles ${ }^{(12)}$. Outro aspecto que pode ser relacionado é a automedicação, prática comum entre os adultos, entretanto, menos aplicada quando se refere à saúde de crianças ${ }^{(13)}$.

Quanto às patologias mais frequentes, este estudo identificou o Impetigo, a Varicela, a Escabiose, a Dermatite de Contato e a Tínea corpus; resultado este que é diferente do perfil apresentado no Brasil e no Mato Grosso. Segundo a Sociedade Brasileira de Dermatologia em 2006, os atendimentos dermatológicos no Brasil tiveram como maior prevalência a acne (14\%); seguida por micose superficial $(8,7 \%)$; transtorno de pigmentação $(8,4 \%)$; ceratose actinea $(5,1 \%)$ e, por fim, a dermatite de contato $(3,9 \%)^{(3)}$. Destaca-se que, no bairro da pesquisa a acne encontra-se em $14^{\circ}$ lugar, presente em $0,51 \%$ dos prontuários. 
Vale ressaltar que o presente estudo foi realizado em uma UBS, que presta atenção primária, de iniciativa pública, resultando em uma demanda que pode ser diferenciada de outros serviços públicos ou privada. Coloca-se também, que na população estudada houve predomínio de pessoas na etapa da infância, impossibilitando o elevado número de prontuários com registro de diagnóstico de acne.

No Mato Grosso, as doenças dermatológicas mais predominantes são acne, transtornos da pigmentação e micoses superficiais. Sendo que, os transtornos de pigmentação são cloasma, hiperpigmentação ou hipercromias, sardas ou efélide e leucodermia, e as micoses superficiais são tínea das unhas, dermatofitose não especificada, pitiríase versicolor e candidíase não especificada $^{(3)}$.

As doenças de pele são fortemente influenciadas por fatores como idade, condições de moradia e higiene e grau de escolaridade. Estes fatores podem ter influenciado na divergência dos resultados apresentados, já que esta pesquisa foi desenvolvida em uma única unidade de saúde, sendo então seus resultados não generalizáveis para outras populações. Das cinco patologias identificadas, três (Impetigo, Escabiose e Tínea corpus) delas têm suas formas de contágio especialmente relacionadas com as condições de higiene. O que nos mostra a possível relação dos índices encontrados com a situação socioeconômica e faixa etária das pessoas envolvidas no estudo. Diante da problemática, a realização de educação em saúde, principalmente com as mães, sobre cuidados de higiene, e a melhora nas condições sanitárias, podem diminuir a disseminação de tais patologias.

Em outro aspecto, a Sociedade Brasileira de Dermatologia( $^{(3)}$ relata a identificação e o processo diagnósticos das doenças dermatológicas relacionadas com a qualificação específica do profissional médico que está realizando as consultas. Na presente pesquisa, os pacientes registrados foram atendidos por médicos clínicos gerais, o que talvez justifique a identificação e registro de, em alguns casos, sinais de doenças de pele, ao invés do diagnóstico propriamente dito.

A varicela, apresentada em segundo lugar neste estudo, está incluída no programa de imunobiológicos especiais do Ministério da Saúde, ou seja, a imunização para varicela não está disponível para toda a população. A vacina contra varicela está indicada para imunocomprometidos, portadores de leucemia, imunocompetentes, profissionais de saúde, pessoas e familiares suscetíveis à doença e que estejam em convívio domiciliar ou hospitalar com pacientes imunocomprometidos, e pessoas suscetíveis à doença que serão submetidas a transplante de órgãos (fígado, rins, coração, pulmão e outros órgãos sólidos)(14).

Entretanto, após a realização de parceria entre o laboratório público e um laboratório privado britânico que possibilitará a produção nacional da vacina tetra viral, a vacina contra varicela será disponibilizada ao Programa Nacional de Imunização (PNI) do Ministério da Saúde a partir de agosto de 2013. Esta medida poderá proporcionar mudanças na atual epidemiologia da doença ${ }^{(15)}$.

No que se refere à relação da prevalência dessas cinco doenças com fatores sazonais, não foram encontrados dados relevantes na literatura, excetuando-se quanto ao Impetigo, que ocorre mais frequentemente no verão ${ }^{(4)}$. Porém, no presente estudo aconteceu em maior quantidade no mês de maio de 2009, o que iria de encontro com o outono. Entretanto, ressalta-se que no Estado Mato Grosso, um estado normalmente com temperatura amenas e altas, as estações se resumem a períodos de chuva e de seca, sendo maio um mês que aborda o final da temporada de chuva e o começo de seca.

O enfermeiro que atua em Programas de Saúde da Família pode trazer importantes contribuições no que se refere às morbidades dermatológicas, especialmente, por meio da consulta de enfermagem, envolvendo a Sistematização da Assistência de Enfermagem, e de educação em saúde voltada para o indivíduo, família e comunidade. O enfermeiro deve, então, obter informações sobre hábitos e comportamentos que podem influenciar no surgimento das doenças dermatológicas, para direcionar o planejamento do cuidado. As prescrições de enfermagem envolvem principalmente instruir o paciente e a família sobre práticas de boa higiene $\mathrm{e}$ utilização adequada da terapêutica medicamentosa.

Para o Impetigo, tais medidas visam evitar a disseminação das lesões de uma área cutânea para outra e de uma pessoa para outra. Para pessoas com Varicela, busca-se, com os cuidados de enfermagem, prevenir infecções secundárias por bactérias. Quanto à Escabiose, o enfermeiro orienta que o paciente deve usar roupas limpas e dormir com roupas de cama recentemente lavadas ${ }^{(4)}$.

Em uma situação que envolva Dermatite de Contato, o enfermeiro tem importante participação na investigação das possíveis causas, além de orientar proteção da pele contra lesões adicionais. No caso de Tínea corpus,deve-se garantir a utilização correta dos fármacos e a proteção contra infecções secundárias por bactérias e/ou Candida ${ }^{(4)}$. 


\section{CONCLUSÃO}

A pesquisa não atingiu todas as suas metas, pois os prontuários em sua maioria não eram preenchidos de forma completa, impossibilitando de se analisar plenamente as patologias dermatológicas.

Sugere-se que a atenção primária de saúde necessita de maior direcionamento para o atendimento de nosologias dermatológicas, visto que grande parte dessas doenças são preveníveis, ressaltando a necessidade de medidas de educação em saúde e saneamento básico. Neste contexto o enfermeiro encontra-se em posição privilegiada, podendo proporcionar o diagnóstico precoce e a prevenção, por meio da realização de um exame físico durante a consulta de enfermagem e de medidas de educação em saúde. Portanto, por meio desta pesquisa, espera-se estimular o enfermeiro a cada vez mais conquistar sua autonomia, por meio do conhecimento e da assistência de qualidade em doenças dermatológicas.

Por fim, colocamos que seria de grande valia a realização de novos estudos para descrever dados epidemiológicos sobre doenças dermatológicas, relacionando com aspectos sociodemográficos e ambientais. Pois, a exemplo deste estudo, que teve como patologias mais frequentes o Impetigo, a Varicela, a Escabiose, a Dermatite de Contato e a Tínea corpus, as doenças dermatológicas são bastante influenciadas por fatores externos.

\section{REFERÊNCIAS}

1. Lopes AC (editor). Tratado de Clínica Médica. $2^{\mathrm{a}}$ ed. São Paulo: Roca; 2009.

2. Rodrigues JG, Costa IMC, Leite R, Soares R. Sociedade Brasileira de Dermatologia. Acervo raro da Sociedade Brasileira de Dermatologia: considerações sobre sua preservação histórica. An. bras. dermatol. [Internet] 2009;84(1) [acesso em 02 fev 2013]. Disponível: http:// dx.doi.org/10.1590/S0365-05962009000100017

3. Lopes LRS, Kundman D, Duarte IAG. Avaliação da frequência de dermatoses no serviço ambulatorial de dermatologia. An. bras. dermatol. [Internet] 2010;85(2) [acesso em 02 fev 2013]. Disponível: http://dx.doi. org/10.1590/S0365-05962010000200024

4. Smeltzer SC, Bare BG, Hinkle JL, Cheever KH. Brunner e Suddart - Tratado de Enfermagem Médico Cirúrgica. $12^{\mathrm{a}}$ ed. Rio de Janeiro: Guanabara Koogan; 2011.

5. Lira ALBC, Sá JD, Nogueira ILA, Medeiros MD, Fernandes MICD, Vitor AF. Integridade da pele em idosos: revisão da literatura segundo as Cartas de promoção da saúde. Cogitare enferm. [Internet] 2012;17(4) [acesso em 02 fev 2013]. Disponível: http://ojs.c3sl.ufpr. br/ojs2/index.php/cogitare/article/view/30389/19664

6. Conselho Federal de Medicina. Define prontuário médico e torna obrigatória a criação da Comissão de Revisão de Prontuários nas instituições de saúde. Resolução CFM n. 1.638, de 09 de agosto de 2002, Brasília, 2002.

7. Dicionário de Língua Portuguesa. Patologia. In Infopédia [Em linha]. Porto: Porto Editora; 2003-2013. [acesso em 02 jun 2013]. Disponível: http://www.infopedia. $\mathrm{pt} /$ lingua-portuguesa/patologia;jsessionid=E38VTIXnyoY09Y45LDJtQ

8. Ministério da Saúde (BR). Conselho Nacional de Saúde. Diretrizes e normas regulamentadoras de pesquisas envolvendo seres humanos. Resolução n. 196, de 10 de outubro de 1996. Brasília, 1996.

9. Ruiz G. Quem usa o Sistema Único de Saúde? Rio de Janeiro: Portal DSS Brasil [Internet] 2012 [acesso em 22 mai 2013]. Disponível: http://dssbr.org/ site $/$ $p=9534 \&$ preview $=$ true

10. Nunes AA, Levorato CD, Mello LM, Silva AS. Fatores associados à procura por serviços de saúde numa perspectiva relacional de gênero. Ciênc. saude colet. [Internet] 2013 [acesso em 22 mar 2013]. Disponível: http://www.cienciaesaudecoletiva.com.br/artigos/ artigo_int.php?id_artigo $=12756$

11. Buchalla AP. As mais vaidosas do mundo. Rev. Veja [Internet] 2003 [acesso em 02 fev 2013]. Disponível: http://veja.abril.com.br/030903/p_058.html

12. Travassos C, Viacava F, Pinheiro R, Brito A. Utilização dos serviços de saúde no Brasil: gênero, características familiares e condição social. Rev Panam Salud Publica [Internet]. 2002;11(5-6) [acesso em 12 jun 2013]. Disponível: http://dx.doi.org/10.1590/S102049892002000500011

13. AlmeidaAR,SanchesMYA, RochaNMA.Automedicação e autoprescrição: um estudo piloto sobre o perfil e os possíveis agentes influenciadores dos consumidores de medicamentos alopáticos da região de São Bernardo do Campo. Encipecom [Internet]. 2003 [acesso em 12 jun 2013] Disponível: http://encipecom.metodista.br/ mediawiki/images/c/c4/Estudo_piloto___Adilson.pdf

14. São Paulo. Centro de Vigilância Epidemiológica. Divisão de Imunização. Divisão de Doenças de Transmissão Respiratória. Imunoprofilaxia para 
varicela. 2010 [acesso em 02 fev 2013]. Disponível: http://www.cve.saude.sp.gov.br/htm/resp/pdf/IF10 VARICELA.pdf

15. Ministério da Saúde (BR). SUS terá vacina contra catapora a partir de 2013. 2012 [acesso em 02 fev 2013]. Disponível: http://portalsaude.saude.gov.br/portalsaude/ noticia/6503/162/sus-tera-vacina-contra-catapora-apartir-de-2013.html 